\title{
Spätabtreibung
}

\section{Was der Gesetzgeber ändern muss}

\begin{abstract}
Im Deutschen Bundestag wird derzeit erneut die Frage der Schwangerschafts-Spätabbrüche diskutiert. CDU-MdB Prof. Dr. Maria Böhmer: „Die Praxis zeigt, dass Kinder nach wie vor wegen einer erwarteten Behinderung abgetrieben werden. Das steht in krassem Gegensatz zum Grundgesetz; dort haben wir in Art. 3 den Satz eingefügt: ,Niemand darf wegen seiner Behinderung benachteiligt werden. “' Warum die erneute Diskussion notwendig geworden ist, beschreibt der Ethikexperte PD Dr. Florian Steger, Erlangen/Nürnberg:
\end{abstract}

— Jährlich werden ca. 120000 Schwangerschaftsabbrüche durchgeführt. Solche Abbrüche sind nur dann straffrei, wenn der gesetzliche Rahmen eingehalten wird; andernfalls liegt ein Straftatbestand vor (§§ 218 und 219d StGB).

Für einen Schwangerschaftsabbruch innerhalb der ersten zwölf Wochen sind nach der sogenannten Fristenregelung ein Beratungsgespräch sowie eine Bedenkzeit von drei Tagen vorgeschrieben. Dabei soll der Abbruch selbst von einem anderen Arzt durchgeführt werden als von dem, der das Beratungsgespräch durchgeführt hat. Innerhalb der ersten zwölf Wochen darf ebenfalls eine Abtreibung vorgenommen werden, wenn der Schwangerschaft ein Straftatbestand nach §§ 176-179 StGB (Vergewaltigung) zugrunde liegt. Allein die sogenannte medizinische Indikation unterliegt keiner Befristung: Demnach ist ein Schwangerschaftsabbruch erlaubt, wenn durch den Abbruch die Gefahr einer schwierigen Beeinträchtigung des körperlichen oder seelischen Gesundheitszustandes der Schwangeren oder gar Gefahr für ihr Leben abzuwenden ist.

Für die schwierigen ethischen Konfliktsituationen des späten Schwangerschaftsabbruchs werden nun neue Gesetze gefordert. Es geht um die zunehmende Zahl der Spätabtreibungen. Die gesetzlichen Bestimmungen des Schwangerschaftsabbruchs aus medizinischer Indikation werden als reformbedürftig angesehen.

Bisher ist in Situationen der Spätabtreibung weder ein ausführliches Beratungsgespräch noch eine mehrere Tage umfassende Latenz bis zur definitiven Entscheidung gefordert. Diese Regelung lässt zu viele ungeklärte Fragen in der Praxis offen. Die bis 1995

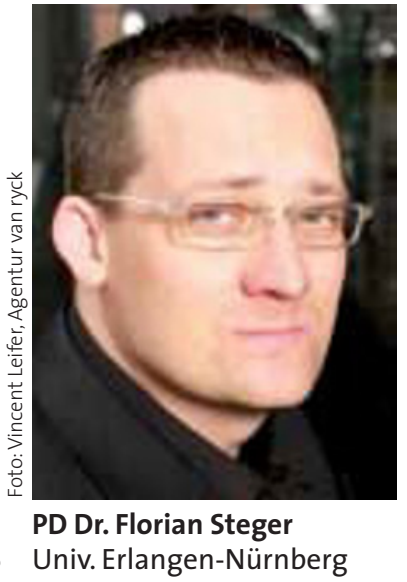
bestehende sog. embryopathische Indikation für den Schwangerschaftsabbruch ging aufgrund eines Urteils des Bundesverfassungsgerichts, das hierin eine Diskriminierung von Behinderten sah, in der „erweiterten medizinischen Indikation“ auf.

Demnach sind Schwangerschaftsabbrüche bis unmittelbar vor der Geburt des Kindes zulässig, wenn eine schwere Schädigung des Kindes und eine erhebliche Beeinträchtigung der Mutter zu erwarten sind; diese Regelung umfasst auch zu erwartende psychische Auswirkungen für die Mutter, die ein behindertes Kind aufziehen muss.

Doch ist in dieser Form das Lebensrecht des Kindes hinreichend berücksichtigt? Nach Art. 1 und 2 GG stehen dem ungeborenen Menschen Würde

\section{Zitat}

„Es ist doch geradezu widersinnig, dass dann, wenn die Schwangerschaft fortgeschritten ist und das Konfliktpotenzial und die Belastung der Frau in dieser Situation noch größer werden, weil das Kind lebensfähig ist, das Schutz- und Beratungskonzept wegfällt. Denn dann sind keine verbindliche Beratung und keine Bedenkzeit mehr gegeben. Die Mutter steht ohne Hilfe da, sie ist auf sich allein gestellt, sie ist allein gelassen. Unsere Auffassung ist: So kann es nicht bleiben, das muss geändert werden." CDU-MdB Prof. Dr. Maria Böhmer und Lebensschutz zu. Dem steht das Selbstbestimmungsrecht der Schwangeren gegenüber. Doch kann die Schwangere bei den derzeitigen Bestimmungen zu einer überlegten Entscheidung für ihr Leben und ihre Gesundheit kommen?

Entsprechend setzt sich die Bundesärztekammer für eine Änderung der Bestimmungen zum späten Schwangerschaftsabbruch ein. Gefordert werden eine verpflichtende ausführliche ärztliche Beratung und eine drei Tage dauernde Latenz zwischen Diagnose und Eingriff. Zudem wird empfohlen, dass sich die Befristung für einen späten Abbruch am Entwicklungsstadium des Kindes orientiert. Hier werden vor allem ab der 23 . Schwangerschaftswoche strenge Kriterien gefordert.

\section{Überlebt das Kind den Abbruch, ist sein Leben zu erhalten}

Die Bestimmung des Entwicklungsstadiums ist auch in der medizinethischen Diskussion ein zentraler Gesichtspunkt, da es neben der Auffassung der absoluten Schutzwürdigkeit des Embryos auch den Begriff der abgestuften Schutzwürdigkeit gibt, der von im Laufe der Entwicklung erworbenen Eigenschaften oder Fähigkeiten abhängig gemacht wird. Besonders schwierig wird die Situation für den Arzt, wenn das Kind beim Abbruch der Schwangerschaft lebend geboren wird: Der Arzt hat dann das Leben dieses Kindes zu erhalten.

- PD Dr. Florian Steger, Institut für Geschichte und Ethik der Medizin, Friedrich-Alexander-Universität Erlangen-Nürnberg, Glückstraße 10, D-91054 Erlangen 